\title{
The New American Joint Commission on Cancer Staging System for Soft Tissue Sarcomas: Splitting versus Lumping
}

\author{
Sam S. Yoon, MD \\ Gastric and Mixed Tumor Service, Department of Surgery, Memorial Sloan Kettering Cancer Center, New York, NY
}

The staging of soft-tissue sarcomas (STS) with the traditional TNM methodology used by the American Joint Commission on Cancer (AJCC) has been problematic for several reasons. First, there are more than 50 different histologic subtypes of STS, all of which have varying epidemiology, clinical features, biology, responses to therapy, and prognoses. The primary reason to group these heterogeneous histologies together is that they are generally thought to arise from mesenchymal tissues, but that is akin to lumping all adenocarcinomas together. Second, STS arise throughout the body, and biological behavior, local recurrence, and distant metastasis often are site-dependent. Finally, STS rarely metastasize to lymph nodes (except for a few subtypes, such as synovial sarcoma, clear cell sarcoma, angiosarcoma, adult rhadomyosarcoma, and epithelioid sarcoma), and thus the $\mathrm{N}$ category of TNM staging is not particularly useful.

Prior editions of the AJCC staging system for STS have compensated for these issues by incorporating histologic grade as part of the T category. ${ }^{1,2}$ Low-grade STS rarely metastasize and thus are considered stage I regardless of the size. The risk of metastasis for intermediate and highgrade tumors increases as tumor size increases, so these tumors are stages II to IIIB depending on size. The most recent, 8th edition of the AJCC staging system for STS corrects one major issue in that it now splits the staging of STS into anatomic sites, with the trunk and extremity now staged separately from other sites, such as the head and neck or retroperitoneum. ${ }^{3}$ Other changes include the following:

\footnotetext{
(C) Society of Surgical Oncology 2018
}

First Received: 21 February 2018; Published Online: 12 March 2018

S. S. Yoon, MD

e-mail: yoons@mskcc.org
1. $\mathrm{T}$ categories have increased from two $(\mathrm{T} 1 \leq 5 \mathrm{~cm}$; $\mathrm{T} 2>5 \mathrm{~cm})$ to four $(\mathrm{T} 1 \leq 5 \mathrm{~cm} ; \mathrm{T} 2>5 \mathrm{~cm}, \leq 10$ $\mathrm{cm} ; \mathrm{T} 3>10 \mathrm{~cm}, \leq 15 \mathrm{~cm} ; \mathrm{T} 4>15 \mathrm{~cm}$ )

2. AJCC prognostic stage groups have changed, including classifying node positive disease as stage IV rather than stage III.

In this issue of Annals of Surgical Oncology, Fisher et al. use the National Cancer Database (NCDB) to analyze more than 26,000 patients with trunk and extremity STS and compare the 7th and 8th editions of the AJCC staging systems. ${ }^{4}$ They determine that separation of the $\mathrm{T}$ categories into four groups and the modification of the AJCC prognostic groups increases the number of patients now classified as stage III and that the overall stage groupings have better discrimination in terms of overall survival. However, dividing tumors $\geq 10 \mathrm{~cm}$ in size into two $\mathrm{T}$ stages (T3 and T4) does not divide those patients into groups with different overall survivals. Classifying nodepositive disease as stage IV rather than stage III increases the percent of patients classified as stage IV in less than $1 \%$ of patients (from 9.8 to $10.6 \%$ ). They also determined that node-positive patients without metastases (N1M0) have an 5 -year overall survival of $33.1 \%$, which was intermediate between stage III patients and stage IV patients.

Fisher et al. should be commended for putting together a large cohort of STS patients and performing a thorough comparison of the 7th and 8th editions of the AJCC staging systems, especially given that the 8th edition has just been published. One primary advantage of this study is the use of the large NCDB, which captures approximately $70 \%$ of the newly diagnosed cancer cases in the United States. The staging modifications in the 8th AJCC staging system were based on single-institution databases and require validation. However, as with most studies of this nature, there are several potential problems. First, the extent and quality of radiologic staging likely varied between institutions. For 
most trunk and extremity sarcomas, the most common site of distant metastasis is the lungs, and we do not know if all patients received high-resolution chest CTs. In addition, some sarcoma subtypes, such as myxoid/round cell liposarcomas, preferentially metastasize to extra-pulmonary sites. ${ }^{5}$ These patients generally need CT scans of the chest, abdomen, and pelvis and possibly even total spine MRIs for complete radiologic staging. ${ }^{6}$ Inadequate radiologic staging may have missed some patients who had synchronous metastatic disease. Second, the extent of surgery, use of neoadjuvant/adjuvant radiation, and systemic therapy varies widely among institutions and may have impacted on pathological staging and survival. We are not told what percent of patients received neoadjuvant therapy, and this therapy can significantly alter the surgical pathology. Finally, the only requirement for follow-up in these patients was that they have at least 90 days of followup. Thus, patients with poor follow-up or patients lost to follow-up after 90 days may skew the overall survival results.

Two additional, important points should be made regarding the staging of patients with STS. While the AJCC 8th edition staging system for STS represents a significant advance in terms of now grouping tumors by location, it continues to disregard histologic subtype. Given there are more than 50 different histologic subtypes, it may be impractical to account for them all in one staging system. Including tumor grade in the $\mathrm{T}$ categories only partially makes up for the varying biology of histologic subtypes. Various nomograms have accounted for the more common histologic subtypes in making predications regarding local and distant recurrence, and it is possible that future editions of the AJCC staging system will include such nomograms following validation. ${ }^{7,8}$ Also, the stage of patients with lymph node metastasis and no distant metastasis (N1M0) continues to change. In the AJCC 6th edition, these patients were stage IV, and in the AJCC 7th edition, these patients were stage III. In the current edition, these patients are now stage IV again. In the Fisher study, N1M0 patients only represented $0.8 \%$ of patients, and the overall survival of these patients suggests that these patients should be stage IIIB.

Niels Bohr, who received the 1922 Nobel Prize in Physics for his work on atomic structures, stated "Prediction is very difficult, especially if it's about the future." Yogi Berra, the often-quoted New York Yankee player and manager, made a similar statement decades later. Overall, the article by Fisher et al. suggests that the 8th edition of the AJCC staging system for STS improves on prior editions. However, there are issues not addressed in the current AJCC staging system that future revisions may need to consider, including neoadjuvant therapy and biomarkers.

\section{REFERENCES}

1. AJCC cancer staing manual. 6th ed. New York: Springer; 2002.

2. AJCC cancer staging manual. 7th ed. New York: Springer; 2010.

3. AJCC cancer staging manual 8th ed. New York: Springer; 2017.

4. Fisher SBC, Feig B, Cormier JN, Hunt KK, Torres KE, Roland CL. Comparative performance of the 7th and 8th editions of the American Joint Commission on Cancer staging systems for soft tissue sarcoma of the trunk and extremities. Ann Surg Oncol. 2018. https://doi.org/10.1245/s10434-018-6378-9.

5. Asano N, Susa M, Hosaka S, Nakayama R, Kobayashi E, Takeuchi $\mathrm{K}$, et al. Metastatic patterns of myxoid/round cell liposarcoma: a review of a 25-year experience. Sarcoma. 2012;2012:345161.

6. Schwab JH, Boland PJ, Antonescu C, Bilsky MH, Healey JH. Spinal metastases from myxoid liposarcoma warrant screening with magnetic resonance imaging. Cancer. 2007;110(8):1815-22.

7. Callegaro DM, Miceli R, Bonvalot S. Development and external validation of two nomograms to predict overall survival and occurrence of distant metastases in adults after surgical resection of localised soft-tissue sarcomas of the extremities: a retrospective analysis. Lancet Oncol. 2016;17(5):671-80.

8. Kattan MW, Leung DH, Brennan MF. Postoperative nomogram for 12-year sarcoma-specific death. $J$ Clin Oncol. 2002;20(3):791-6. 